\title{
Transanal Hemorrhoidal Dearterialization With Doppler Arterial Identification Versus Classic Hemorrhoidectomy: A Retrospective Analysis of 270 Patients
}

\author{
Vincenzo Consalvo ${ }^{1,2}$, Francesca D’Auria ${ }^{3}$, Vincenzo Salsano ${ }^{4}$ \\ ${ }^{1}$ Clinique Clementville, Università degli Studi di Salerno, Fisciano, Italy; ${ }^{2}$ Clinique Clemetville, Montpellier, France ${ }^{3}$ AOU San Giovanni di \\ Dio e Ruggi d’Aragona, Salerno, Italy; ${ }^{4}$ Clinique Clementville, Clinique du Parc, Montpellier, France
}

Purpose: Despite the minimally invasive nature of transanal hemorrhoidal dearterialization (THD) with Doppler arterial identification procedures, hemorrhoidectomy is still considered the gold standard procedure for hemorrhoidal disease. However, the classical techniques of hemorrhoidectomy have a high rate of postoperative complications. The main purpose of this study is to demonstrate the efficacy and complications of these techniques used for grades II and III hemorrhoids.

Methods: A retrospective (case-control) study was carried out from January 2009 to May 2014, and all patients undergoing surgical procedures for hemorrhoidal disease in two French clinics were considered. Application of inclusion and exclusion criteria identified 270 eligible patients (163 undergoing Doppler THD and 107 treated with Milligan Morgan hemorrhoidectomy). Statistical analysis was calculated considering immediate postoperative complications, functional results, chronic complications, and recurrences.

Results: Analysis of primary outcomes showed a significant difference between the 2 groups concerning postoperative pain, which had a lower rate in THD $(\mathrm{P}=0.0001)$ and in postoperative bleeding $(\mathrm{P}=0.02)$ than hemorrhoidectomy. However, long-term follow-up at three years showed a superior rate of recurrence in the THD group $(\mathrm{P}=0.009)$.

Conclusion: The THD technique is a safe and effective procedure for grades II and III hemorrhoids, has lower rates of post-operative pain and bleeding, and allows faster hospital discharge; however, it also shows a higher rate of recurrence at three years of follow-up.

Keywords: THD; Transanal haemorrhoid dearterialisation; Milligan Morgan technique; Complications; Recurrence

\section{INTRODUCTION}

Doppler-guided hemorrhoidal artery ligation is a validated procedure for grades II and III hemorrhoids. In 1995, Morinaga et al. [1] described for the first time the transanal hemorrhoidal dearte-

Received: July 11, 2017 - Accepted: September 4, 2017

Correspondence to: Vincenzo Consalvo, M.D.

Clinique Clementville, Università degli Studi di Salerno, via Principe di

Carignano, 4 Mercato san Severino (SA), Italy

Tel: +393279792206, Fax: +33-467024112

E-mail: vincenzoconsa@hotmail.it

ORCID code: https://orcid.org/0000-0003-1671-2259

(C) 2019 The Korean Society of Coloproctology

This is an open-access article distributed under the terms of the Creative Commons Attribution NonCommercial License (http://creativecommons.org/licenses/by-nc/4.0) which permits unrestricted noncommercial use, distribution, and reproduction in any medium, provided the original work is properly cited. rialization (THD) with Doppler arterial identification technique. In 2011, Ratto et al. [2] reported the first cases of THD with Doppler arterial identification using a standard kit (THD Doppler, THD s.p.a., Reggio Emilia, Italy), which is a more standardized procedure than the original THD.

Despite the minimally invasive nature of Doppler THD procedures, there is a lack of supporting evidence around this technique. In fact, hemorrhoidectomy is still considered the gold standard procedure for high-degree hemorrhoidal disease. However, the classical techniques of hemorrhoidectomy (e.g., Milligan Morgan [3], Ferguson [4]) do have a high rate of complications in the postoperative period or during long-term follow-up $[5,6]$. In this study, we described our results with and performed a comparison between the 2 techniques. 


\section{METHODS}

\section{Objectives}

The main purpose of this study is to demonstrate the efficacy and complications of the 2 techniques used for grades II and III hemorrhoids.

\section{Ethical and administrative information}

The study was carried out in accordance with The Code of Ethics of the World Medical Association (Declaration of Helsinki) for experiments involving humans and was approved by a local ethical committee of University of Salerno (IRB No. IRB00000871). The retrospective study was registered in the public registry on http://www.researchregistry.com, with the number 2757.

All patients were informed of the possible risk associated with the surgical procedures and accepted this risk with informed consent. The authors declare that there will no communication of personal data to third parties to respect patient privacy. All patients completed the anonymous form of acceptance for publication for a scientific purpose and storage of data in a database.

The article was conducted according to Strengthening The Reporting of Observational Studies in Epidemiology (STROBE) guidelines [7].

\section{Key features, eligibility criteria, procedure descriptions, and follow-up}

A retrospective (case-control) methodology was chosen for this study. Our general database was reviewed from January 2009 to May 2014, and all patients undergoing surgical procedures for hemorrhoidal disease in 2 French clinics were selected.

Inclusion criteria were as follows: patients of any age, ethnicity, or sex treated for grade II or III hemorrhoidal disease with or without rectal prolapse. Grades I and IV were not included because we adopted conservative management for grade I and always perform Milligan Morgan hemorrhoidectomy for grade IV, for which THD is not optimal. Participants were divided into 2 groups: (1) Those receiving THD with Doppler arterial identification were called the "case group," while (2) those receiving hemorrhoidectomy were part of the "control group."

Exclusion criteria were grade IV hemorrhoidal disease and patients with recurrence of any grade of hemorrhoidal disease.

Diagnosis of primary hemorrhoid was established by examination and anoscopy or proctoscopy. Classification of hemorrhoidal disease was graded according to the Goligher classification [8]. Diagnosis of recurrence was established when symptoms did not improve or worsened [9] during the 3-year follow-up.

All surgical procedures were performed by the same 2 surgeons to reduce procedural bias and to provide a criterion of selection for THD and classical hemorrhoidectomy. One surgeon was an expert in the THD method (>300 procedures), while the other was an expert in Milligan Morgan hemorrhoidectomy ( $>500$ procedures). All patients were placed under general anesthesia, and a presacral block with infiltration of $20 \mathrm{~mL}$ of ropivacaine $2 \%$ was further administered in both procedures.

THD with Doppler arterial identification was performed using the THD kit (THD Italy, Corregio, Italy). The surgical procedure described by Ratto et al. [2] was followed. Mucopexy was also performed in all THD procedures.

Hemorrhoidectomy was performed according to the Milligan and Morgan technique.

All patients were discharged the day of surgery (ambulatory protocol) and were prescribed anti-inflammatory drugs.

Follow-up was based on one visit in the first postoperative week and one visit per month for three months. Long-term follow-up was achieved with one phone call per year to all patients or by patient request for consultation with the doctor. All contacted patients suspected of recurrence or other anal morbidities were examined, and diagnosis was confirmed or denied.

Loss to follow-up was defined as missing at least one of the scheduled consultations and/or inability to contact the patient by phone.

\section{Definition of outcomes}

The primary outcomes were post-operative pain, postoperative bleeding, and postoperative fecal incontinence (does not include patients with previous fecal incontinence). Current pain level was evaluated using the brief pain inventory (BPI), a pain assessment tool based on the Brief Pain questionnaire [10]. Fecal incontinence was measured with the Fecal Incontinence Quality of life tool [11].

Secondary outcomes were recurrence rates of hemorrhoidal disease and/or chronic complications, such as anal stenosis, anal fissure, or unhealed wound at 3-year follow-up.

\section{Definition of complications}

Postoperative anal pain: subjective sensation of pain starting on 1 day after surgical intervention. Postoperative bleeding: continuous or intermittent bleeding since 1 day after surgical intervention. Postoperative fecal incontinence: objective impossibility to contract internal and external sphincters, confirmed by anorectal tests. Recurrence of hemorrhoid: clinical diagnosis or symptoms that did not improve or worsened [9] by the 3-year follow-up. Anal stenosis: clinical diagnosis.

\section{Statistical analysis}

All data were analyzed with InStat by GraphPad ver. 3.10 (GraphPad Software Inc., La Jolla, CA, USA). A Mann-Whitney test was used to match quantitative variables (e.g., age). Fisher exact test was used for analysis of the contingency tables of postoperative and chronic complications between the case and control groups. A 2-tailed t-test was used to match means of unpaired data (BMI, BPI score, and fecal continence quality of life score). 


\section{RESULTS}

A total of 321 patients was considered eligible after application of inclusion and exclusion criteria. Nevertheless, loss to follow-up was not insignificant: 11 patients informed us they would be followed in a public hospital after operation and were excluded from the study. Eight patients missed follow-up at 1 month, 10 missed follow-up at 2 months, and another 10 missed the 3-month visit; lastly, 12 patients were not able to be contacted by phone.

Of 321 potentially eligible patients, only 270 completed all follow-up visits and were confirmed and included in the study. Loss to follow-up represented $15.8 \%$ of the total population.

The THD group included 163 patients, while the control group had only 107. In the THD group, 92 individuals were classified as grade II and 71 as grade III, while 62 in the control groups were grade II and 45 were grade III. There was not a statistically significant difference between the 2 groups concerning age or BMI, as shown in Table 1.

Analysis of primary outcomes (Table 2) showed a significant difference between the 2 groups in postoperative pain, which was lower in THD than hemorrhoidectomy $(\mathrm{P}=0.0001)$, and in postoperative bleeding $(\mathrm{P}=0.02)$. No significant difference was found in postoperative fecal incontinence rate $(P=0.39)$. See Table 2 for further details.

On the contrary, functional outcome analysis (Table 3 ) did not show a significant difference in BPI pain severity index $(\mathrm{P}=0.29)$ or BPI pain interference $(\mathrm{P}=0.43)$, while analysis of fecal continence quality of life score was not calculated because of the small number of cases.

Lastly, long-term complications were analyzed, and no statistical difference was found in anal stenosis, anal fissure, or unhealed

Table 1. Patient characteristics

\begin{tabular}{lccc}
\hline Characteristic & $\begin{array}{c}\text { THD } \\
(\mathrm{n}=163)\end{array}$ & $\begin{array}{c}\text { Hemorrhoidectomy } \\
(\mathrm{n}=107)\end{array}$ & P-value \\
\hline Sex, male : female & $95: 68$ & $45: 62$ & \\
Age $(\mathrm{yr})$ & $37.3 \pm 18.5$ & $35.1 \pm 18.8$ & 0.34 \\
Body mass index $\left(\mathrm{kg} / \mathrm{m}^{2}\right)$ & $26.3 \pm 6.1$ & $27.0 \pm 7.2$ & 0.39 \\
\hline
\end{tabular}

Values are presented as number or mean \pm standard deviation. THD, transanal hemorrhoidal dearterialization. wound at three years from surgical intervention ( 0 total complications in both groups), but hemorrhoid recurrence was higher ( $\mathrm{P}$ $=0.009)$ in the THD group $(\mathrm{n}=24,14.7 \%)$ compared to the Milligan Morgan group ( $\mathrm{n}=5,4.67 \%)$ (Table 4). All 24 patients in THD group underwent successful reoperation with the THD technique and experienced no complications in the postoperative period, while 4 patients in the control group accepted the Milligan Morgan reoperation and 1 patient refused further surgery.

\section{DISCUSSION}

Treatment of hemorrhoids is still debated. While hemorrhoidectomy is the gold standard procedure, there are several drawbacks and complications associated with this procedure. Some of these complications impact quality of life (anal fissure, unhealed anal wound), while others could be invalidating (fecal incontinence) [11]. For this reason, researchers are looking for alternative procedures, such as Doppler THD. Ratto et al. [12] showed an absence of important complications, which are represented mainly by anal tenesmus (13\% of cases). Hoyuela et al. [13] reported a 2-year prospective study and concluded that THD is a safe and almost painless technique that offers very good results in the control of hemorrhoidal symptoms. Infantino et al. [14] reported similar results to this multicentric prospective trial.

However, there is further evidence [15] showing similar pain perception, postoperative complications, and long-term outcomes between THD and hemorrhoidectomy.

In our series, we found a lower rate of overall postoperative complications, especially postoperative pain $(9.8 \%, \mathrm{P}=0.0001)$ and bleeding $(6.58 \%, \mathrm{P}=0.02)$, compared to Milligan Morgan hemorrhoidectomy. Although most patients were discharged on

Table 3. Functional outcomes results

\begin{tabular}{lccc}
\hline Variable & THD & Hemorrhoidectomy & P-value \\
\hline BPI pain severity & $0.33 \pm 0.55$ & $0.71 \pm 1.4$ & 0.29 \\
BPI pain interference & $0.51 \pm 0.11$ & $0.75 \pm 1.2$ & 0.43 \\
Fecal continence QoL score & $\mathrm{NG}$ & 1 & $\mathrm{NG}$ \\
\hline
\end{tabular}

Values are presented as mean \pm standard deviation.

THD, transanal hemorrhoidal dearterialization; BPI, brief pain inventory; QoL, quality of life; NG, not given.

Table 2. Primary outcomes analysis

\begin{tabular}{|c|c|c|c|c|c|}
\hline Variable & $\begin{array}{c}\text { THD } \\
(n=163)\end{array}$ & $\begin{array}{l}\text { Hemorrhoidectomy } \\
(n=107)\end{array}$ & P-value ${ }^{a}$ & Odds ratio & $95 \% \mathrm{Cl}$ \\
\hline Postoperative anal pain & $16(9.8)$ & $67(62.6)$ & $0.0001^{\star}$ & 0.07 & $0.034-0.124$ \\
\hline Postoperative bleeding & $11(6.58)$ & $17(15.9)$ & $0.02^{*}$ & 0.38 & $0.172-0.855$ \\
\hline Postoperative fecal incontinence & $0(0)$ & $1(0.9)$ & 0.39 & 0.21 & $0.009-5.417$ \\
\hline
\end{tabular}

Values are presented as number (\%).

THD, transanal hemorrhoidal dearterialization; $\mathrm{Cl}$, confidence interval.

${ }^{*} \mathrm{P}<0.05$, significant difference. ${ }^{\mathrm{F}}$ Fisher exact test. 
Table 4. Chronic complications at 3 years of follow-up

\begin{tabular}{|c|c|c|c|c|c|}
\hline Variable & THD $(n=163)$ & Hemorrhoidectomy $(n=107)$ & P-value ${ }^{a}$ & Odds ratio & $95 \% \mathrm{Cl}$ \\
\hline Recurrence of hemorrhoid & $24(14.7)$ & $5(4.67)$ & $0.009^{*}$ & 3.52 & $1.3-9.54$ \\
\hline Anal stenosis, fistula, others & $0(0)$ & $0(0)$ & $N G$ & NG & $N G$ \\
\hline
\end{tabular}

Values are presented as number (\%).

THD, transanal hemorrhoidal dearterialization; $\mathrm{Cl}$, confidence interval; NG, not given.

${ }^{*} \mathrm{P}<0.05$, significant difference. ${ }^{\mathrm{a}}$ Ficher exact test.

the day (ambulatory) of the procedure, but we had to prolong recovery for patients with anal pain or bleeding. However, in most cases, conservative management with anti-inflammatory drugs and metronidazole was sufficient for both complications, while 1 case of uncontrollable anal pain and 2 cases of anal bleeding required correction one day after the initial surgery.

In summary, functional data did not reveal any statistically significant difference between the 2 techniques. Therefore, when pain was present, there was no difference in pain severity of interference between the 2 techniques. It was not possible to perform statistical analysis for fecal incontinence because only 1 case was observed in the hemorrhoidectomy series.

Our data after a 3-year follow-up period confirmed the vast majority of previous author results: recurrence of hemorrhoid is higher with the THD technique (14.7\%) compared to hemorrhoidectomy (4.67\%). This suggests that THD is less reliable in the long-term, especially as long-term complication rate was $0 \%$ in both techniques.

Considering the overall compliance of patients and the reductions in postoperative complications and pain in THD technique, we suggest that recurrence after a certain period of time be an acceptable risk to avoid postoperative pain and experience a faster recovery. Furthermore, when recurrence occurs, classical hemorrhoidectomy remains an option.

This was not a randomized controlled trial, and results should be interpreted considering a potential source of bias. Loss to follow-up was discrete in our series, and other results could interfere with the statistics considered. Further studies with a longer follow-up are necessary.

In conclusion, the THD technique is a safe and effective procedure for grades II and III hemorrhoids. Compared to classical Milligan Morgan hemorrhoidectomy, the THD method has lower rates of postoperative pain and bleeding, allowing for faster hospital discharge. Long-term results showed inferior reliability in recurrence rate between the methods, while there was no difference in chronic complications. Additional larger studies are necessary to confirm our results.

\section{CONFLICT OF INTEREST}

No potential conflict of interest relevant to this article was reported.

\section{REFERENCES}

1. Morinaga K, Hasuda K, Ikeda T. A novel therapy for internal hemorrhoids: ligation of the hemorrhoidal artery with a newly devised instrument (Moricorn) in conjunction with a Doppler flowmeter. Am J Gastroenterol 1995;90:610-3.

2. Ratto C, Giordano P, Donisi L, Parello A, Litta F, Doglietto GB. Transanal haemorrhoidal dearterialization (THD) for selected fourth-degree haemorrhoids. Tech Coloproctol 2011;15:191-7.

3. Agbo SP. Surgical management of hemorrhoids. J Surg Tech Case Rep 2011;3:68-75.

4. Ferguson JA, Heaton JR. Closed hemorrhoidectomy. Dis Colon Rectum 1959;2:176-9.

5. Qarabaki MA, Mukhashavria GA, Mukhashavria GG, Giorgadze NG. Circular vs. three-quadrant hemorrhoidectomy for end-stage hemorrhoids: short- and long-term outcomes of a prospective randomized trial. J Gastrointest Surg 2014;18:808-15.

6. Konsten J, Baeten CG. Hemorrhoidectomy vs. Lord's method: 17year follow-up of a prospective, randomized trial. Dis Colon Rectum 2000;43:503-6.

7. von Elm E, Altman DG, Egger M, Pocock SJ, Gøtzsche PC, Vandenbroucke JP, et al. The Strengthening the Reporting of Observational Studies in Epidemiology (STROBE) statement: guidelines for reporting observational studies. J Clin Epidemiol 2008; 61:344-9.

8. Goligher JC, Leacock AG, Brossy JJ. The surgical anatomy of the anal canal. Br J Surg 1955;43:51-61.

9. Tiernan JP, Hind D, Brown SR. Evaluating the efficacy of treatments for haemorrhoids: time for a standardized definition of recurrence? Colorectal Dis 2013;15:1449-50.

10. Keller S, Bann CM, Dodd SL, Schein J, Mendoza TR, Cleeland CS. Validity of the brief pain inventory for use in documenting the outcomes of patients with noncancer pain. Clin J Pain 2004; 20:309-18.

11. Rockwood TH, Church JM, Fleshman JW, Kane RL, Mavrantonis C, Thorson AG, et al. Fecal incontinence quality of life scale: quality of life instrument for patients with fecal incontinence. Dis Colon Rectum 2000;43:9-16.

12. Ratto C, Parello A, Veronese E, Cudazzo E, D’Agostino E, Pagano $\mathrm{C}$, et al. Doppler-guided transanal haemorrhoidal dearterialization for haemorrhoids: results from a multicentre trial. Colorectal Dis 2015;17:O10-9.

13. Hoyuela C, Carvajal F, Juvany M, Troyano D, Trias M, Martrat A, 


\section{Coloproctology vincenzo consalvo, et al.}

et al. HAL-RAR (Doppler guided haemorrhoid artery ligation with recto-anal repair) is a safe and effective procedure for haemorrhoids. Results of a prospective study after two-years follow-up. Int J Surg 2016;28:39-44.

14. Infantino A, Bellomo R, Dal Monte PP, Salafia C, Tagariello C, Tonizzo CA, et al. Transanal haemorrhoidal artery echodoppler ligation and anopexy (THD) is effective for II and III degree haemorrhoids: a prospective multicentric study. Colorectal Dis 2010;12:804-9.

15. De Nardi P, Capretti G, Corsaro A, Staudacher C. A prospective, randomized trial comparing the short- and long-term results of doppler-guided transanal hemorrhoid dearterialization with mucopexy versus excision hemorrhoidectomy for grade III hemorrhoids. Dis Colon Rectum 2014;57:348-53. 